\title{
On the connections between solar and stellar dynamo models
}

\author{
Laurène Jouve ${ }^{1,2}$ and Rohit Kumar ${ }^{1,2}$ \\ ${ }^{1}$ Université de Toulouse, UPS-OMP, Institut de Recherche en Astrophysique et Planétologie, \\ 31028 Toulouse Cedex 4, France \\ ${ }^{2}$ CNRS, Institut de Recherche en Astrophysique et Planétologie, 14 avenue Edouard Belin, \\ 31400 Toulouse, France
}

\begin{abstract}
We here discuss the various dynamo models which have been designed to explain the generation and evolution of large-scale magnetic fields in stars. We focus on the models that have been applied to the Sun and can be tested for other solar-type stars now that modern observational techniques provide us with detailed stellar magnetic field observations. Mean-field flux-transport dynamo models have been developed for decades to explain the solar cycle and applications to more rapidly-rotating stars are discussed. Tremendous recent progress has been made on 3D global convective dynamo models. They do not however for now produce regular flux emergence that could be responsible for surface active regions and questions about the role of these active regions in the dynamo mechanism are still difficult to address with such models. We finally discuss 3D kinematic dynamo models which could constitute a promising combined approach, in which data assimilation could be applied.
\end{abstract}

Keywords. dynamo, magnetic fields, convection, rotation, numerical simulations

\section{Introduction}

The magnetic activity of our Sun has been monitored for centuries through the recording of the number of spots regularly emerging at the solar surface. The first telescope observations date back from the beginning of the 17th century when the attention of famous astronomers like Galileo and Thomas Harriott started to be drawn by those spots present at the surface of our star. It was only much later that those spots were associated with the presence of a magnetic field with a Zeeman signature discovered in a sunspot by Hale(1908). We now know that the solar magnetic field is not only responsible for the periodic appearance of sunspots at the surface but is also the triggering mechanism for powerful flares and coronal mass ejections that may strongly interact with our own terrestrial magnetosphere. Understanding the various consequences of the solar magnetic field requires to investigate its origins. Quite rapidly, the dynamo mechanism was invoked to be the process through which the Sun would maintain its magnetic field. A need to develop models and later numerical simulations appeared inevitable, to fully understand how the plasma flows would organize themselves in the solar interior to produce a magnetic field with a 22-yr period and all the associated phenomena at the solar photosphere and higher in its atmosphere. Two different approaches were adopted for the simulation of the solar dynamo, as we will develop later: mean-field dynamo models (Moffatt(1978); Krause \& Rädler(1980)) which deal only with the large-scale magnetic field, assuming some parameterization of the underlying small-scale turbulence and magnetism and 3D global models which solve the full set of magnetohydrodynamical (MHD) equations, allowing to self-consistently produce the flow and magnetic field structures which will non-linearly interact (see reviews of Miesch \& Toomre(2009) and Brun et al.(2015)). 
Various models and associated numerical simulations have thus been developed to explain the characteristics of the solar magnetic field, with different levels of success (see review by Charbonneau(2014)). Despite all theses efforts, the community still does not have at its disposal a global model of the Sun which reproduces self-consistently the cyclic appearance of sunspots at the solar surface, showing the extraordinary complexity of our nearest star and the difficulties to model it accurately. Tremendous progress has been made recently on the observations of magnetic field on stars other than the Sun. It was indeed known from observations of the emissions in $\mathrm{Ca}$ lines $\mathrm{H}$ and $\mathrm{K}$ at the Mount Wilson Observatory that some stars possess chromospheric activity cycles on time-scales of years to decades (Baliunas et al.(1995)). Even if the relationship with a magnetic cycle is not necessarily direct (See et al.(2016)), it implied that other stars could also exhibit cyclic reversals of their magnetic field. Zeeman-Doppler Imaging (ZDI) now provides a way to monitor the topology and intensity of stellar magnetic fields in time. This tomographic technique is capable of reconstructing large-scale magnetic field topologies at stellar surfaces by inverting a series of spectropolarimetric observations (Donati \& Brown(1997)). This technique revealed fascinating features about the magnetism of cool stars (Folsom et al.(2016), Morin et al.(2010)). It was for example found that fully convective stars appear to mostly host strong dipolar fields (Gregory et al.(2012)) or that rapidly rotating partly convective stars tend to produce strong toroidal structures (Morin et al.(2010), Petit et al.(2008)). Repeated observations of individual targets have now even revealed polarity reversals (Fares et al.(2009), Morgenthaler et al.(2011), Boro Saikia et al.(2016)), suggesting the presence of magnetic cycles.

We are thus now reaching a point where models which have been applied to the Sun for years or decades should now be tested on other cool stars and compared to stellar observations. We discuss the connections between solar and stellar dynamo models in 4 different sections. In section 1, 2D mean field dynamo models are discussed. In section 2 , we rapidly review the great recent progress of $3 \mathrm{D}$ global dynamo models. In the last 2 sections, we focus on magnetic flux emergence: section 3 is dedicated to the detailed simulations of the particular process of flux emergence through the stellar interior and the production of spots and section 4 discusses the use of a combined approach: 3D kinematic dynamo models.

\section{Flux transport dynamo models : applying the solar paradigm to stellar dynamos}

\subsection{The Babcock-Leighton dynamo model applied to the Sun}

The past few decades have seen the advent of multidimensional numerical simulations to better understand the generation and the intricate non-linear evolution of the solar magnetic field. In order to model and understand the large-scale magnetic field, a useful approach has been to make use of the mean field dynamo theory. Among the various mean field dynamo models, the Babcock-Leighton (Babcock(1961), Leighton(1969)) flux transport dynamo models have recently been massively applied to the Sun. In the Babcock-Leighton (BL) model, the toroidal magnetic field owes its origin to the differential rotation at play in the stellar convection zone, while the poloidal field originates from the decay of active regions popping up at the stellar surface with a particular field strength and tilt angle. If we then add a large-scale meridional circulation, whose role is to advect the magnetic field concentrations inside the convection zone, the model is called a flux-transport model. This model demonstrated great success at reproducing some solar observations such as the 11-yr cycle, the mid-latitude activity belt, the 

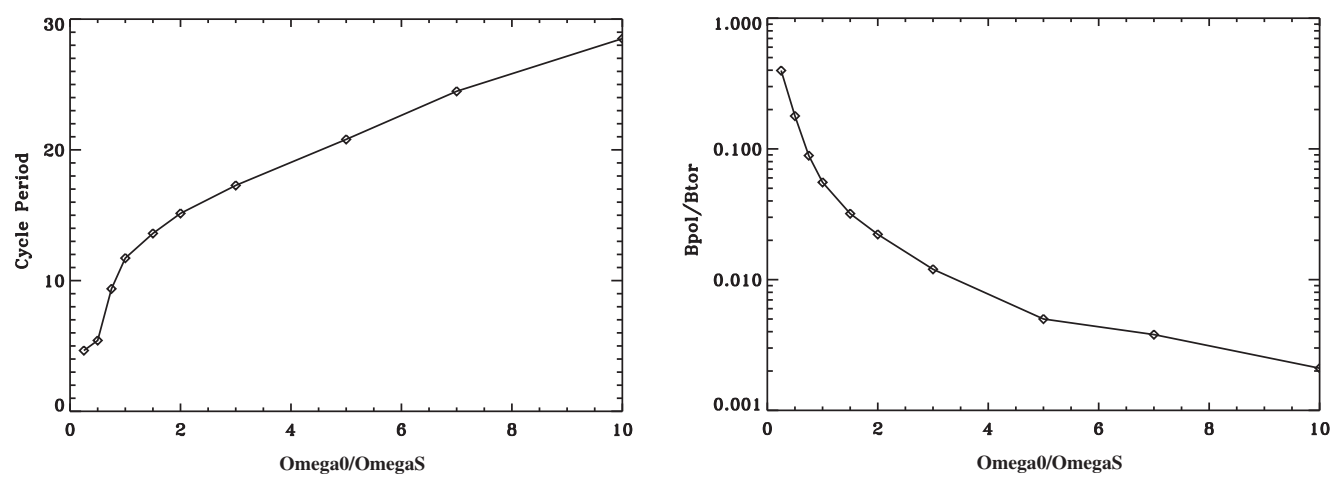

Figure 1. Period of the dynamo cycle (left panel) and poloidal to toroidal field ratio (right) as a function of the stellar rotation rate, normalized to the solar rotation rate. From simulations of Jouve et al.(2010).

phase relationship between toroidal and poloidal fields or the equatorward propagation of sunspot emergence (see Living Review by Charbonneau(2005)). These models even start to be used now to give tentative predictions of the next solar cycle, using data assimilation techniques which have been commonly used in meteorology for decades (Jouve et al.(2011), Dikpati \& Anderson(2014), Hung et al.(2015)). It is then legitimate to ask if such models can be applied to other stars and lead to the same agreement with available observations. In particular, the observations seem to indicate that rapidly rotating solar-type stars tend to possess shorter magnetic cycles and exhibit stronger toroidal field components. Such a study was performed by Jouve et al.(2010) and we here summarize their findings.

\subsection{The Babcock-Leighton dynamo model applied to the rapidly-rotating stars}

The peculiarity of flux-transport models is that they indeed produce a magnetic field regularly reversing its polarity, with a cycle period extremely sensitive to the meridional flow amplitude $v_{0}$. Dikpati \& Charbonneau(1999) or Jouve \& Brun(2007) report scalings such as $P_{c y c} \propto v_{0}^{-0.83}$. It is thus necessary to have a hint of the amplitude of the meridional circulation in stars other than the Sun, which is a difficult problem. Indeed, already in the Sun are the characteristics of the meridional flow poorly constrained by helioseismology (see review by Gizon et al.(2010)). In other stars, this flow is not observed at all. However, 3D MHD numerical simulations of rapidly rotating stars do exist, showing that the meridional flow amplitude decreases with increasing rotation rate (Ballot et al.(2007), Brown et al.(2008), Augustson et al.(2012)) with a typical scaling such that $v_{0} \propto \Omega_{0}^{-0.45}$. The same 3D numerical simulations also tend to show that the meridional flow becomes more and more multicellular as the rotation rate increases.

Applying BL flux-transport dynamo models with faster rotation and thus slower meridional circulation, Jouve et al.(2010) thus found that the magnetic cycle period in those stars should be much longer than suggested by the observations. Figure 1 illustrates those results by showing the period of the mean-field dynamo cycle with respect to the stellar rotation rate (left panel). The period is clearly increased when the rotation is increased because of the decrease in amplitude of the meridional flow speed. We note however that more toroidal field is produced compared to the poloidal field when the rotation is increased (right panel), in agreement with the spectropolarimetric observations of Petit et al.(2008) for example. It is however still possible to reconcile the models with observations by reducing the dependency of the magnetic cycle period with respect to $v_{0}$, for example, by invoking other transport processes such as turbulent diffusion or 
magnetic pumping (Guerrero \& de Gouveia Dal Pino(2008), Do Cao \& Brun(2011), Hazra et al.(2014)). Nevertheless, applying a model well-calibrated for the Sun to other stars here proved this model had to be severely modified to fit with stellar observations.

\section{3D global MHD models}

\subsection{Hydrodynamical models}

As already stated above, the full set of MHD equations is solved in 3D MHD global models in spherical geometry. Those models are then necessarily much more costly than 2D mean-field models, but have the decisive advantage of self-consistently computing the flows and magnetic fields which will non-linearly interact. Tremendous progress has been done in the past decade on these 3D models and several properties about stellar convection, large-scale flows and dynamos have been found to be quite robust in the various simulations performed with such models (see review in Brun et al.(2015)). For instance, they all show that the differential rotation profile is directly linked to the Rossby number of the simulation, which is a measure of the importance of the inertia term compared to the Coriolis term in the Navier-Stokes equation. Indeed, it was found by several authors that anti-solar differential rotation state (slow equator, fast poles) occur at large Rossby number whereas solar-like differential rotation state (fast equator, slow poles) occurs at low Rossby number (see synthesis of large number of numerical simulations by Gastine et al.(2014)). Those calculations also agree on the fact that fast rotation (i.e. low Rossby numbers) implies a decrease in amplitude of the meridional circulation (as stated above) and a more complex structure, with several circulation cells appearing both in latitude and radius (Featherstone \& Miesch(2015)). This again may have strong consequences on flux-transport dynamo models (Jouve \& Brun(2007)).

\subsection{Magnetic models of low-mass stars}

As far as magnetic fields are concerned in those 3D MHD models, robust features have also been recovered by various groups. In particular, a wide variety of dynamo behaviours are found, from steady to irregular to well-defined cyclic magnetic activity. For low-mass stars (spectral type M), numerical simulations tend to demonstrate the ordering role of the Coriolis force, also seen in planetary dynamos (Christensen \& Aubert(2006)). More specifically, when the Rossby number is increased, the magnetic field switches from being mostly dipolar to mostly multipolar. However, it has also been found that the low Rossby number regime could maintain both a dipolar solution and a multipolar solution depending on the initial magnetic conditions. This interesting bistability was also seen in observations (Morin et al.(2010)) where two stars with very similar rotation rates and masses (thus probably similar Rossby numbers) exhibit very different magnetic fields (strong and dipolar for one and weak and multipolar for the other). However, with a stronger stratification, this bistable behaviour seems to disappear in simulations. More computations are thus needed to further investigate this property. For instance, a recent simulation of a fully convective star by Yadav et al.(2015) with a reasonable degree of stratification (a density ratio of 150) was shown to possess both large-scale (mostly dipolar) and small-scale magnetic fields. A ZDI reconstruction was then applied to the simulation to see how well this analysis technique was able to recover the magnetic field content. As expected, the large-scale strong polar spot was perfectly recovered but not the smaller-scale features, which represent most of the magnetic flux in the simulation. 


\subsection{Magnetic models of solar-type stars}

Simulations of solar-type stars with high rotation rates were also performed, showing strong belts of toroidal field in the convection zone which could start to undergo cyclic reversals as the level of turbulence is increased (Brown et al.(2011), Käpylä et al.(2013)). Some Maunder minima-like periods were even found in some simulations of $\mathrm{F}$ stars (Augustson et al.(2013)). It is still not entirely clear in those models possessing cyclic reversals what sets the cycle period. From published results, the meridional circulation amplitude does not seem to play a key role in establishing the time scale for the magnetic cycle, contrary to what is assumed in a Babcock-Leighton flux-transport dynamo model. However, those 3D dynamos do not produce spots at their surfaces and may thus be difficult to reconcile with BL models. We will come back on this in the next section.

Most of the simulations cited above do not possess a tachocline and a stable layer beneath. Only recently have some simulations been performed considering a tachocline and comparisons with convective shells having similar properties (Guerrero et al.(2016)). It is reported that a tachocline helps to organise the magnetic field by building strong concentrations of large-scale field. However, the influence of the tachocline on the cyclic behaviour of the solution still needs to be clarified.

\section{Flux emergence : what properties of solar flux emergence coud be applied to other stars?}

\subsection{Spot formation in $3 D$ global models}

In both mean-field dynamo models and global MHD models discussed above, the process of magnetic flux emergence through the stellar convection zone is crucial. In the Sun, it is the strong toroidal structures built at the base of the convection zone which are assumed to be unstable to a buoyancy instability and rise through the convection zone to produce sunspots (Parker(1955)). This particular step is crucial for BL models since the source of poloidal field is directly linked to the presence of active regions. In 3D models, the strong toroidal structures built in rapidly-rotating stars can become buoyant (Nelson et al.(2013), Fan \& Fang(2014)) but rarely rises all the way to the top of the computational domain and those models consequently do not produce spots. It is thus still an open question if we can really rely on spotless dynamo models to reproduce what could happen in stars or in the Sun. The particular step of flux emergence being potentially important for the whole dynamo mechanism, detailed numerical simulations of such a process are thus needed. It has to be noted that other theories exist which do not rely so strongly on the presence of strong toroidal structures built in the tachocline and then becoming unstable. Some authors (Stein \& Nordlund(2012), Brandenburg et al.(2013)) have argued that local flux concentrations by convective motions or by instabilities appearing in very strongly stratified zones could also lead to the formation of active regions in the Sun. We here concentrate only on the first picture of flux tubes rising from the base of the convection zones where they are produced, to the surface where they emerge as spots.

\subsection{Simulations of flux emergence in the Sun and possible applications to other stars}

Numerous numerical simulations of flux emergence have been performed for the Sun for which detailed observations of active region formation and evolution exist (see Living Review by $\operatorname{Fan}(2004)$ ). An illustration of a numerical simulation of a buoyant loop rising in a convective shell is shown in Fig. 2. These simulations are able to reproduce several features of solar active regions: their morphology, their tilt angle due both to the twist of 


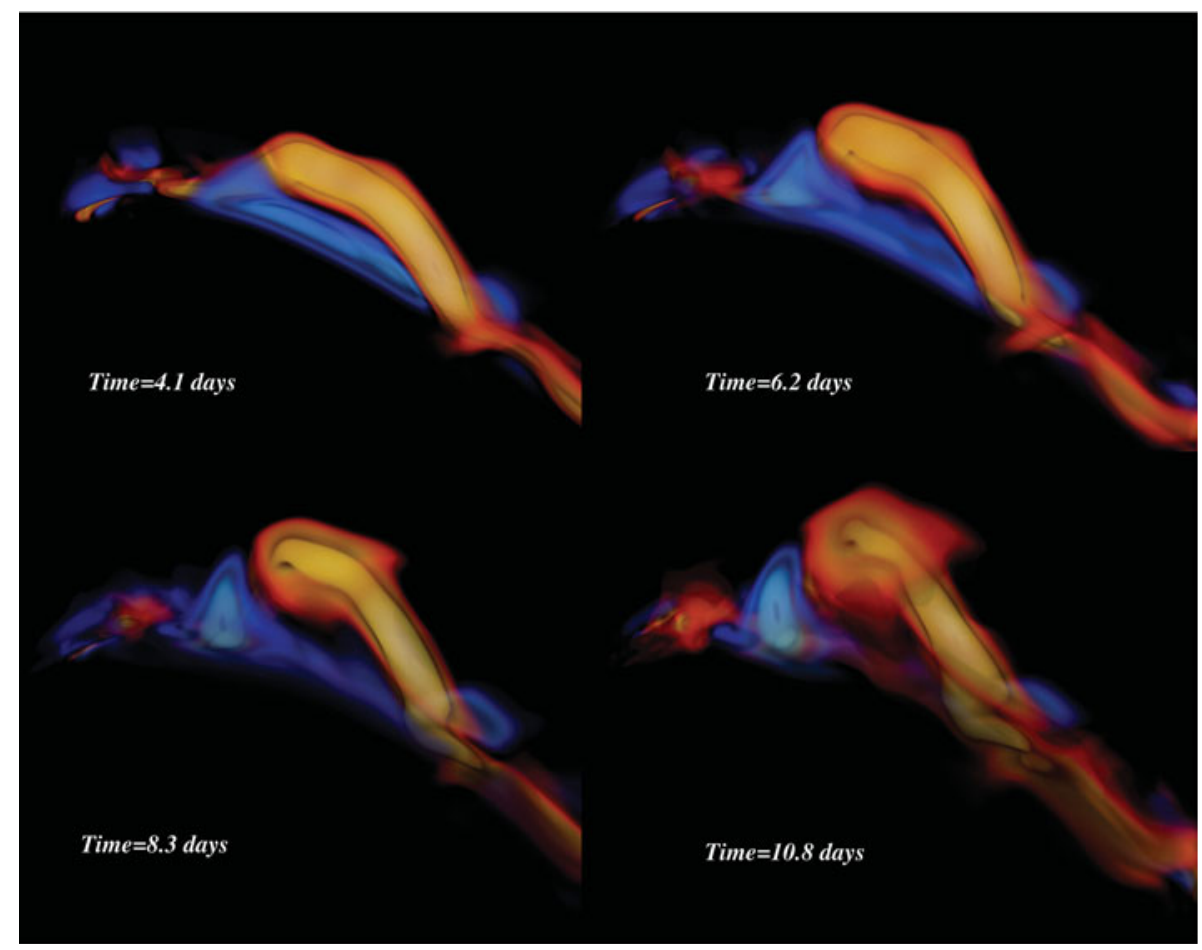

Figure 2. Volume rendering of the toroidal magnetic field of a loop rising through a convective layer. From simulations of Jouve et al.(2013).

the field lines and the Coriolis force acting on the rising tubes, the asymmetry between the leading and trailing spots or the interplay with the local convective flows. However, very few investigations have been conducted on similar processes of flux emergence in other stars. We note that thin flux tube calculations have been conducted by for giants (Holzwarth \& Schüssler(2001)) and rapidly rotating stars (Holzwarth et al.(2006)), and simulations of thin flux tubes evolving in a fully convective star performed recently by Weber \& Browning(2016). In this last article, they were particularly interested in the latitude of emergence of starspots, which they found to be strongly related to the stellar rotation and to the thermodynamical characteristics of their thin flux tubes.

Indications exist today of spots on other stars, with various degrees of surface coverage and magnetic fluxes (see review by Berdyugina(2005)). These properties have strong implications for potential eruptive activity on those stars and consequences on surrounding planets. Some properties found in simulations of large-scale flux emergence in the Sun could be easily applied to other stars. In particular, the rise trajectory of field concentrations from the base of the convection zone to the surface is strongly influenced by the Coriolis force, with a tendency of flux tubes in a fast rotating environment to rise parallel to the rotation axis and thus emerge at high latitudes. We thus expect rapidly rotating stars to exhibit spots at high latitude, which seem to be in reasonable agreement with observations. The typical size of active regions is then another question which could be addressed through numerical simulations of flux emergence in a convective domain. Is this size determined by the mean size of convective cells (which is different in stars rotating at different rates) or by the typical length-scale of the buoyancy instability, this question remains to be answered in detail. 


\section{A combined approach: 3D kinematic dynamo models}

\subsection{Modelling buoyancy in kinematic models}

The Babcock-Leighton mechanism relies on the decay of active regions to reverse the polar field. In such kinds of models, the production of bipolar magnetic regions (BMR) at the stellar surface is thus essential to sustain dynamo action. This mechanism is supported by various solar observations: firstly, BMR are seen to continuously emerge and diffuse at the solar surface with a sufficient flux to be able to reverse the polar field. Secondly, correlations exist between the strength of the BL mechanism in one cycle and the amplitude (i.e. the number of sunspots) of the next cycle (Dasi-Espuig et al.(2010)). However, as stated before, the 3D global models exhibiting dynamo cycles do not produce well-defined BMRs and thus can not address the question of the role of the BL mechanism in the full solar and stellar dynamo loop. Motivated by solar observations and lack of self-consistent models to produce spots at the solar/stellar surface, some research groups started to develop an intermediate approach between the 2D BL flux-transport dynamo models which take for granted that the BL mechanism is the main player in the dynamo loop and 3D global models which do not capture the spot-producing mechanism. In this type of models, called 3D kinematic dynamo models, a velocity field is prescribed (as in 2D mean-field kinematic models) and a "buoyancy algorithm" is implemented to extract toroidal flux concentrations produced at the base of the convection zone and to translate them into BMRs at the surface. Two different models were developed so far, differing in this "buoyancy algorithm": Miesch \& Dikpati(2014) and then Miesch \& Teweldebirhan(2016) use a version of the "double-ring" algorithm (Durney(1997), Nandy \& Choudhuri(2001)) to place BMRs at the solar surface in response to the dynamogenerated field at the base of the convection zone. Yeates \& Muñoz-Jaramillo(2013) use a more self-consistent model in which an additional velocity is applied to the toroidal structures to make them rise to the surface.

\subsection{Promising models for data assimilation?}

Kumar \& Jouve (in prep.) are currently building a model similar to the one of Yeates \& Muñoz-Jaramillo(2013), by adapting the pseudo-spectral code MagIC (Wicht(2002)). For their simulation, they use an initial magnetic field which has two components: an equatorially antisymmetric strong toroidal field at the base of the convection zone, and a relatively weaker large-scale poloidal field. The magnetic buoyancy along with a helical flow give rise to tilted BMRs at the outer surface; eight such BMRs are illustrated in Figure 3(a). Note that the tilt angle is large for the BMRs at higher latitudes. Later, the decay and dispersal of these tilted BMRs lead to the polarity reversal of the large-scale polar field (see Figure 3(b)). These models are very appealing since they provide a way to directly test the ability of BMRs to reverse the polar fields, taking into account the main ingredients thought to be important in the dynamo mechanism: the meridional circulation and magnetic diffusion at the solar surface and the building of toroidal flux at the base of the convection zone by the differential rotation. Moreover, several prescriptions from 3D models of flux emergence can be easily reintroduced in these models, such as the field strength-dependent rise time of the flux tubes or the latitude of emergence which will be modified when faster rotation is considered. Such models will thus be able to be designed not only for the Sun but for other stars which produce spots. Finally, those models will be very well-adapted to the application of data assimilation techniques to predict the future solar activity. Data assimilation has been used for decades for weather forecasting on Earth. The idea is to combine time-dependent models and data available over a certain time interval so that the misfit between the outputs of the model and 

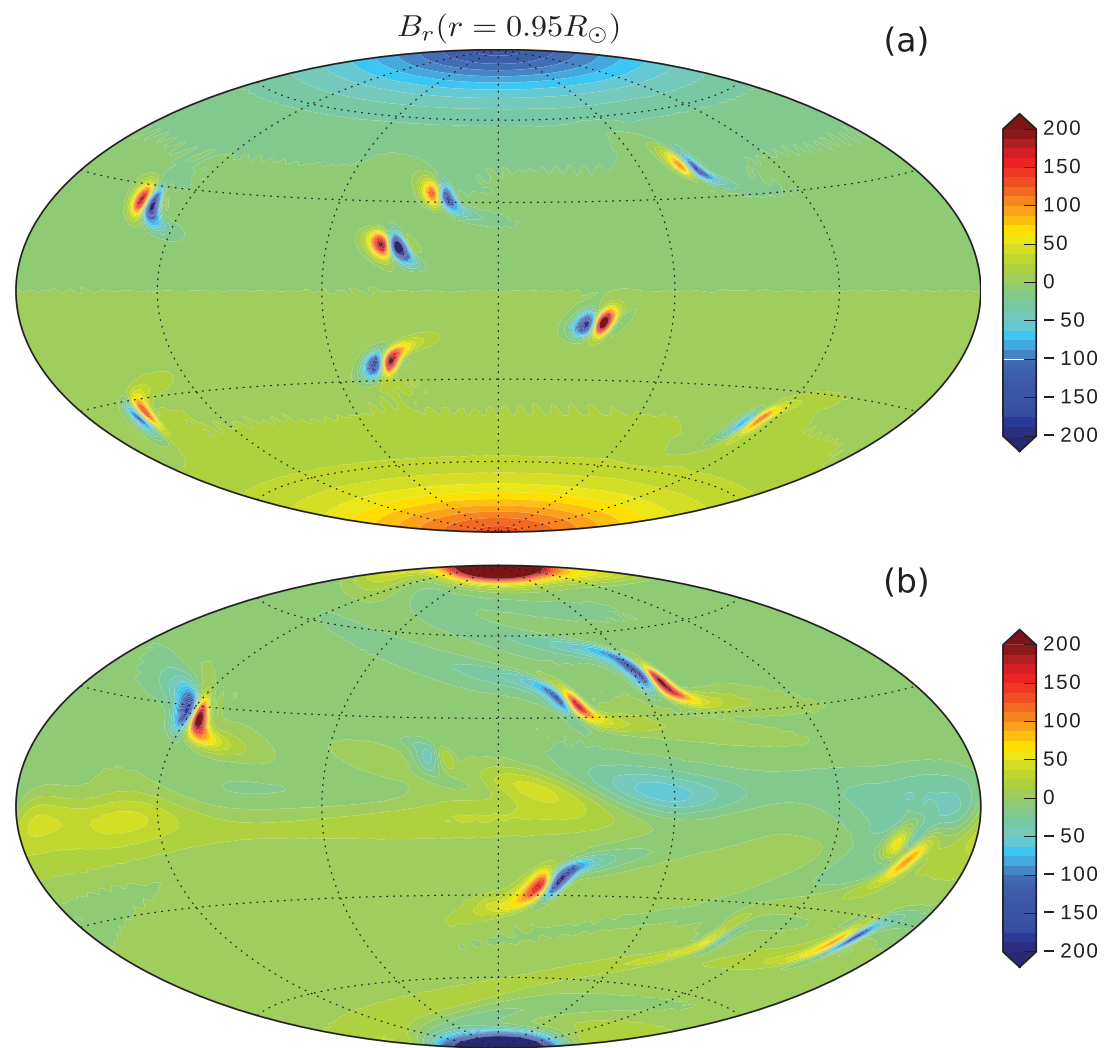

Figure 3. Snapshots of the non-dimensional radial magnetic field $\left(B_{r}\right)$ at the surface corresponding to the radius $r=0.95 R_{\odot}$ showing (a) the initial large-scale poloidal field along with eight tilted BMRs, and (b) after the polarity reversal, the poloidal field and BMRs of the next cycle (note the polarity switch). From Kumar \& Jouve (in prep.).

the observational data is minimized. After this "learning phase", the model is optimized to fit previous observations and is ready to provide forecast for the future behavior of the system. Attempts to apply data assimilation to solar physics have already been performed in 2D mean-field dynamo models (Hung et al.(2015), Dikpati et al.(2016) for the most recent) but only on synthetic data. The direct comparisons between BMRs produced in $3 \mathrm{D}$ kinematic models and solar active regions, between surface observations of the meridional flow and the one used in the simulation and between the observed and simulated polar flux could be very promising for the application of data assimilation in such models. This will be the subject to future work.

\section{Conclusion}

We rapidly reviewed here some features of dynamo models used for the Sun and the possibilities to apply such models to other stars. We are indeed reaching a fascinating time when detailed observations of magnetic fields on stars other than the Sun become available and where confronting solar models to stellar observations can be performed. Some attempts have been made to apply Babcock-Leighton flux-transport dynamo models to rapidly-rotating solar like stars. In those models, the magnetic cycle period is typically set by the amplitude of the meridional flow, which is still not well known in the solar interior and completely unknown for other stars. However, 3D global models of 
rapidly-rotating stars tend to show that the meridional flow speed decreases when the rotation rate is increased, thus producing a longer magnetic cycle period for more rapidly rotating stars. This does not seem to agree with observations of chromospheric activity cycles. Moreover, recent 3D dynamo simulations which exhibit cyclic reversal do not find any correlations between the meridional flow amplitude and the magnetic cycle period, contrary to what is expected in flux-transport models. We note however that those 3D more complete and self-consistent models do not produce a regular emergence of bipolar magnetic regions as seen in the Sun. This may be only a question of time before these global models do produce starspots but in the meantime, a useful approach is to consider $3 \mathrm{D}$ kinematic dynamo models. They represent an intermediate approach between the 2D dynamo models where the BL mechanism is crucial for the dynamo and 3D global models which do not capture the spot-producing mechanism. Application of this kind of models to other stars would also be possible and particularly interesting, to investigate the effects of faster rotation, larger convection zones on the production of spots and on the BL mechanism. We anticipate that those models will be very promising for the application of data assimilation techniques, with the aim to produce reliable forecast of future solar (and possibly stellar?) activity.

\section{Acknowledgements}

LJ and RK acknowledge support from the Indo-French Centre for the Promotion of Advanced Research (CEFIPRA), via the collaborative project grant 5004-B.

\section{References}

Augustson, K. C., Brown, B. P., Brun, A. S., Miesch, M. S., \& Toomre, J. 2012, Astrophysical Journal, 756, 169

Augustson, K. C., Brun, A. S., \& Toomre, J. 2013, Astrophysical Journal, 777, 153

Babcock, H. W. 1961, Astrophysical Journal, 133, 572

Baliunas, S. L., Donahue, R. A., Soon, W. H., et al. 1995, Astrophysical Journal, 438, 269

Ballot, J., Brun, A. S., \& Turck-Chièze, S. 2007, Astrophysical Journal, 669, 1190

Berdyugina, S. V. 2005, Living Reviews in Solar Physics, 2, 8

Boro Saikia, S., Jeffers, S. V., Morin, J., et al. 2016, Astronomy 83 Astrophysics, 594, A29

Brandenburg, A., Kleeorin, N., \& Rogachevskii, I. 2013, Astrophysical Journal, Letters, 776, L23

Brown, B. P., Browning, M. K., Brun, A. S., Miesch, M. S., \& Toomre, J. 2008, Astrophysical Journal, 689, 1354-1372

Brown, B. P., Miesch, M. S., Browning, M. K., Brun, A. S., \& Toomre, J. 2011, Astrophysical Journal, 731, 69

Brun, A. S., Browning, M. K., Dikpati, M., Hotta, H., \& Strugarek, A. 2015, Space Science Reviews, 196, 101

Charbonneau, P. 2005, Living Reviews in Solar Physics, 2, 2

Charbonneau, P. 2014, Annual Review of Astron and Astrophys, 52, 251

Christensen, U. R., \& Aubert, J. 2006, Geophysical Journal International, 166, 97

Dasi-Espuig, M., Solanki, S. K., Krivova, N. A., Cameron, R., \& Peñuela, T. 2010, Astronomy ES Astrophysics, 518, A7

Dikpati, M., \& Charbonneau, P. 1999, Astrophysical Journal, 518, 508

Dikpati, M., \& Anderson, J. L. 2014, AGU Fall Meeting Abstracts

Dikpati, M., Anderson, J. L., \& Mitra, D. 2016, Astrophysical Journal, 828, 91

Guerrero, G., \& de Gouveia Dal Pino, E. M. 2008, Astronomy \& Astrophysics, 485, 267

Do Cao, O., \& Brun, A. S. 2011, Astronomische Nachrichten, 332, 907

Donati, J.-F., \& Brown, S.F. 1997, Astronomy \& Astrophysics, 326, 1135

Durney, B. R. 1997, Astrophysical Journal, 486, 1065

Fan, Y. 2004, Living Reviews in Solar Physics, 1, 1 
Fan, Y., \& Fang, F. 2014, Astrophysical Journal, 789, 35

Fares, R., Donati, J.-F., Moutou, C., et al. 2009, Monthly Notices of the Royal Astronomical Society, 398, 1383

Featherstone, N. A., \& Miesch, M. S. 2015, Astrophysical Journal, 804, 67

Folsom, C.P., Petit, P., Bouvier, J., et al. 2016, Monthly Notices of the Royal Astronomical Society, 457, 580

Gastine, T., Yadav, R. K., Morin, J., Reiners, A., \& Wicht, J. 2014, Monthly Notices of the Royal Astronomical Society, 438, L76

Gizon, L., Birch, A. C., \& Spruit, H. C. 2010, Annual Review of Astron and Astrophys, 48, 289

Gregory, S. G., Donati, J.-F., Morin, J., et al. 2012, Astrophysical Journal, 755, 97

Guerrero, G., \& de Gouveia Dal Pino, E. M. 2008, Astronomy \& Astrophysics, 485, 267

Guerrero, G., Smolarkiewicz, P. K., de Gouveia Dal Pino, E. M., Kosovichev, A. G., \& Mansour, N. N. 2016, Astrophysical Journal, 819, 104

Hale, G. E. 1908, Astrophysical Journal, 28, 315

Hazra, S., Passos, D., \& Nandy, D. 2014, Astrophysical Journal, 789, 5

Holzwarth, V., \& Schüssler, M. 2001, Astronomy \& Astrophysics, 377, 251

Holzwarth, V., Mackay, D. H., \& Jardine, M. 2006, Monthly Notices of the Royal Astronomical Society, 369, 1703

Hung, C. P., Jouve, L., Brun, A.-S., Fournier, A., \& Talagrand, O. 2015, EGU General Assembly Conference Abstracts, 17, 10832

Jouve, L. \& Brun, A. S. 2007, Astronomy \& Astrophysics, 474, 239

Jouve, L., Brown, B. P., \& Brun, A. S. 2010, Astronomy \& Astrophysics, 509, A32

Jouve, L., Brun, A. S., \& Talagrand, O. 2011, Astrophysical Journal, 735, 31

Jouve, L., Brun, A. S., \& Aulanier, G. 2013, Astrophysical Journal, 762, 4

Käpylä, P.J., Mantere, M.J., Cole, E., Warnecke, J., \& Brandenburg, A. 2013, Astrophysical Journal, 778,41

Krause, F. \& Rädler, K.-H. 1980, Organic Photonics and Photovoltaics

Leighton, R. B. 1969, Astrophysical Journal, 156, 1

MacGregor, K. B. \& Charbonneau, P. 1997, Astrophysical Journal, 486, 484

Miesch, M. S., \& Toomre, J. 2009, Annual Review of Fluid Mechanics, 41, 317

Miesch, M. S. \& Dikpati, M. 2014, Astrophysical Journal, Letters, 785, L8

Miesch, M. S. \& Teweldebirhan, K. 2016, Advances in Space Research, 58, 1571

Moffatt, H. K. 1978, Cambridge, England, Cambridge University Press, 1978. 353 p.,

Morgenthaler, A., Petit, P., Morin, J., et al. 2011, Astronomische Nachrichten, 332, 866

Morin, J., Donati, J.-F., Petit, P., et al. 2010, Monthly Notices of the Royal Astronomical Society, 407, 2269

Nandy, D. \& Choudhuri, A. R. 2001, Astrophysical Journal, 551, 576

Nelson, N. J., Brown, B. P., Brun, A. S., Miesch, M. S., \& Toomre, J. 2013, Astrophysical Journal, 762,73

Nelson, N. J., Brown, B. P., Sacha Brun, A., Miesch, M. S., \& Toomre, J. 2014, Solar Physics, 289,441

Parker, E. N. 1955, Astrophysical Journal, 122, 293

Parker, E. N. 1993, Astrophysical Journal, 408, 707

Petit, P., Dintrans, B., Solanki, S. K., et al. 2008, Monthly Notices of the Royal Astronomical Society, 388,80

See, V., Jardine, M., Vidotto, A.A., et al. 2016, Monthly Notices of the Royal Astronomical Society, 462, 4442

Stein, R. F. \& Nordlund, Å. 2012, Astrophysical Journal, Letters, 753, L13

Weber, M. A. \& Browning, M. K. 2016, Astrophysical Journal, 827, 95

Yadav, R. K., Christensen, U. R., Morin, J., et al. 2015, Astrophysical Journal, Letters, 813, L31

Wicht, J. 2002, Physics of the Earth and Planetary Interiors, 132, 281

Yeates, A. R., \& Muñoz-Jaramillo, A. 2013, Monthly Notices of the Royal Astronomical Society, 436,3366 